\title{
Low-Level Laser Therapy for Fibromyalgia: A Systematic Review and Meta-Analysis
}

Shu-Wei Yeh, MD1, Chien-Hsiung Hong, MD1, Ming-Chieh Shih, MD², Ka-Wai Tam, MD, PhD'-6, Yao-Hsien Huang, MD ${ }^{4,7,8}$, and Yi-Chun Kuan, MD ${ }^{2-4,7-9}$

From: ${ }^{1}$ School of Medicine, Taipei Medical University, Taipei, Taiwan; 'Institute of Epidemiology and Preventive Medicine, College of Public Health, National Taiwan University; ${ }^{3}$ Cochrane Taiwan, Taipei Medical University, Taipei, Taiwan; ${ }^{4}$ Center for EvidenceBased Health Care, Taipei Medical University - Shuang Ho Hospital, New Taipei City, Taiwan; 'Division of General Surgery, Department of Surgery, School of Medicine, College of Medicine, Taipei Medical University, Taipei, Taiwan: ${ }^{6}$ Division of General urgery, Department of Surgery, Shuang Ho Hospital, Taipei Medical University, New Taipei

City, Taiwan; ${ }^{7}$ Department of Neurology, Shuang Ho Hospital,

Taipei Medical University, New

Taipei City, Taiwan; ${ }^{8}$ Department of Neurology, School of Medicine, College of Medicine, Taipei Medical University, Taipei, Taiwan; ' ${ }^{2}$ Department of Neurology, Taipei Neuroscience Institute, Taipei Medical University, Taipei, Taiwan

Address Correspondence: Yi-Chun Kuan, MD

Department of Neurology, Taipei Medical University-Shuang Ho Hospital, 291 Zhongzheng Rd, Zhonghe District, New Taipei City, 23561, Taiwan

E-mail: yckuang2@gmail.com

Disclaimer: Shu-Wei Yeh and Chien-Hsiung Hong contributed equally to this study. There was no external funding in the preparation of this manuscript. Conflict of interest: Each author certifies that he or she, or a member of his or her immediate family, has no commercial association (i.e., consultancies, stock ownership, equity interest, patent/licensing arrangements, etc.) that might pose a conflict of interest in connection with the submitted manuscript.

Manuscript received: 09-27-2018 Revised manuscript received: 10-06-2018

Accepted for publication: 11-05-2018

Free full manuscript: www.painphysicianjournal.com
Background: Fibromyalgia is a chronic disorder characterized by widespread pain and tenderness. Low-level laser therapy (LLLT), an emerging nonpharmacological treatment, has been used for relieving musculoskeletal or neuropathic pain.

Objective: The objective of this review and meta-analysis was to determine the efficacy of LLLT on patients with fibromyalgia.

Study Design: This study involved systematic review and quantitative meta-analysis of published randomized controlled trials (RCTs).

Setting: This study examined all RCTs evaluating the effect of LLLT on fibromyalgia.

Methods: We performed a systematic review and meta-analysis of RCTs evaluating the effect of LLLT on patients with fibromyalgia. PubMed, EMBASE, and the Cochrane Library were searched for articles published before August 2018. RCTs meeting our selection criteria were included. The methodological quality of the RCTs was evaluated according to the Cochrane risk-for-bias method. Review Manager version 5.3 was used to perform the meta-analysis. The primary outcomes were the total scores on the Fibromyalgia Impact Questionnaire (FIQ), pain severity, and number of tender points. The secondary outcomes were changes in fatigue, stiffness, anxiety, and depression. Standardized mean difference (SMD), 95\% confidence intervals (CI), and P values were calculated for outcome analysis.

Results: We identified 9 RCTs that included 325 fibromyalgia patients undergoing LLLT or placebo laser treatment with or without an exercise program. The meta-analysis showed that patients receiving LLLT demonstrated significantly greater improvement in their FIQ scores (SMD: 1.16; $95 \% \mathrm{Cl}, 0.64-1.69)$, pain severity (SMD: $1.18 ; 95 \% \mathrm{Cl}, 0.82-1.54)$, number of tender points (SMD: $1.01 ; 95 \% \mathrm{Cl}, 0.49-1.52$ ), fatigue (SMD: $1.4 ; 95 \% \mathrm{Cl}, 0.96-1.84$ ), stiffness (SMD: $0.92 ; 95 \% \mathrm{Cl}, 0.36-1.48)$, depression (SMD: $1.46 ; 95 \% \mathrm{Cl}, 0.93-2.00)$, and anxiety (SMD: 1.46 ; $95 \% \mathrm{Cl}, 0.45-2.47)$ than those receiving placebo laser. Furthermore, when compared with the standardized exercise program alone, LLLT plus the standardized exercise program provided no extra advantage in the relief of symptoms. On the other hand, the results of the only RCT using combined LLLT/LED phototherapy showed significant improvement in most outcomes except for depression when compared to placebo. When compared with pure exercise therapy, combined LLLT/LED phototherapy plus exercise therapy had additional benefits in reducing the severity of pain, number of tender points, and fatigue.

Limitations: There were some limitations in this review, mostly because of the low-to-middle methodological quality of the selected studies; for example, there was no clear allocation process and only patients were blinded in most studies. In addition, one study used per-protocol analysis with a $20 \%$ loss to follow-up. On the other hand, the differences in laser types, energy sources, exposure times, and associated medication status in these studies may have resulted in some heterogeneity.

Conclusions: Our results provided the most up-to-date and relevant evidence regarding the effects of LLLT in fibromyalgia. LLLT is an effective, safe, and well-tolerated treatment for fibromyalgia.

Key words: Low-level laser therapy, fibromyalgia, meta-analysis, FIQ, pain, tender points, exercise

Pain Physician 2019: 22:241-254 
ibromyalgia is a chronic disorder characterized by widespread pain and tenderness. Patients with fibromyalgia often suffer from fatigue, sleep disturbance, and memory problems (1). Neurological complaints such as paresthesia, blurred vision, numbness, and weakness are also commonly seen $(1,2)$. Its estimated prevalence is $2.1 \%$ to $5.3 \%$ in the general population, with women experiencing more severe symptoms. This syndrome typically occurs in middle-aged adults, but it can develop in any age group, including childhood, adolescence, as well as in the elderly (1). Although the cause of fibromyalgia is uncertain, central nervous system sensitization is considered to be its major pathogenesis. External factors such as infection, trauma, and stress may precipitate it $(1,2)$. No curative treatment for fibromyalgia is available thus far. A combination of pharmacological and nonpharmacological treatments is generally recommended for adequate symptom relief (1). The US Food and Drug Administration approved duloxetine (Cymbalta), milnacipran (Savella), and pregabalin (Lyrica) for treating fibromyalgia. Duloxetine and milnacipran help control pain levels by changing some of the brain neurotransmitters (serotonin and norepinephrine), whereas pregabalin blocks the overactivated neurons involved in pain transmission. Physical exercise and cognitive behavior therapy are the nonpharmacological options with stronger evidence of efficacy in fibromyalgia $(3,4)$. Other interventional approaches with lower levels of evidence include occipital nerve stimulation, lidocaine infusion, and hyperbaric oxygen therapy (4).

Low-level laser therapy (LLLT) is an emerging, noninvasive alternative treatment with some efficacy in relieving musculoskeletal or neuropathic pain and improving the quality of life (5-10). The mechanism is believed to involve photochemical reactions, which alter cell membrane permeability, increase messenger RNA buildup, and lead to cell proliferation. The light emitted during LLLT reacts with cytochrome c oxidase, a respiratory enzyme in mitochondria, and increases adenosine triphosphate (ATP) production and reduces reactive oxygen species levels; this helps reduce cell inflammation and death (11). Some randomized controlled trials (RCTs) have investigated the efficacy of LLLT for fibromyalgia. However, the results have been inconsistent, with small sample sizes. Therefore, we conducted a comprehensive systematic review and meta-analysis of the effectiveness of LLLT in fibromyalgia, aiming to contribute to evidence-based decision-making regard- ing the use of LLLT in fibromyalgia. On the other hand, since exercise therapies or physical therapies have been proven to be beneficial to patients with fibromyalgia, we also used meta-analysis to compare the effectiveness of a standardized exercise program plus LLLT with a standardized exercise program alone.

\section{Methods}

\section{Selection Criteria}

We reviewed RCTs evaluating the efficacy of LLLT for fibromyalgia. We included trials that (a) compared the results of LLLT and placebo laser therapy in patients with fibromyalgia; (b) described the inclusion and exclusion criteria for patient selection; and (c) reported power, wavelength, and laser exposure duration. We excluded trials that used a class IV laser because laser class $\leq$ IIIB is considered therapeutic, whereas a class IV laser causes tissue destruction. Furthermore, trials with laser treatment focusing only on one joint or a specific region of the body were excluded.

\section{Search Strategy and Study Selection}

We searched PubMed, EMBASE, and the Cochrane Library for studies on fibromyalgia. The following MeSH terms and Boolean operator were used: fibromyalgia AND (laser OR low-level laser OR photobiomodulation OR phototherapy). The "Related Articles" option in PubMed was used to broaden the search. We applied no language restrictions. The final search was performed in August 2018. We selected studies on the basis of the titles and abstracts meeting the selection criteria. The systematic review described here was accepted by PROSPERO, the online international prospective register of systematic reviews of the National Institute for Health Research (CRD42017079531).

\section{Data Extraction}

Two authors (SWY and $\mathrm{CHH}$ ) independently selected RCTs and extracted the relevant details: number, age, and gender of participants; inclusion and exclusion criteria; laser strategies; and outcome parameters. The individually-recorded information of both reviewers was compared, and a third reviewer (YCK) resolved any discrepancies.

\section{Methodological Quality Appraisal}

The 3 aforementioned reviewers independently evaluated the methodological quality of the RCTs according to the Cochrane risk-for-bias method (12). 
Several domains were evaluated: allocation generation and concealment; blinding of patients, personnel, and outcome assessor; incomplete outcome data (intentionto-treat or per-protocol); and loss to follow-up rate.

\section{Outcome Assessment}

To logically and clearly perform the meta-analysis, the outcome assessment comprised 2 sections. One section pooled data from RCTs comparing LLLT with placebo to evaluate the benefits of LLLT. The other section pooled data from RCTs comparing LLLT plus standardized exercise with standardized exercise alone in order to investigate whether applying the additional laser to exercise therapies provides more benefits than exercise alone.

In each section, we evaluated 3 primary outcomes, namely improvement in the total Fibromyalgia Impact Questionnaire [FIQ] scores (13), severity of pain, and number of tender points; and 4 secondary outcomes, namely improvement in fatigue, stiffness, anxiety, and depression. The improvement in pain severity was assessed by extracting the score of the subitem "pain" from FIQ (0-10), using a 5-point Likert scale $(0=$ none, $1=$ mild, $2=$ moderate, $3=$ severe, and $4=$ extreme), or by using a visual analog scale (VAS) in $\mathrm{cm}$. As for the definition of tender points, points that were reported by patients as being painful were regarded as tender points; additional tender points were more rigorously defined if patients felt pain at pressure less than or equal to $2.6 \mathrm{kgf} / \mathrm{cm}^{2}$ while subject to an increasing pressure of $0.1 \mathrm{kgf} / \mathrm{s}$ via placement of an apparatus perpendicular to the point to be evaluated (14). Improvements in fatigue, stiffness, anxiety, and depression were assessed using the subitem score of "fatigue" on the FIQ (0-10) or on the Likert scoring system for grading, "stiffness" on the FIQ and "morning stiffness" on the Likert scoring system, "anxiety" on the FIQ, and "depression" on the $\mathrm{FIQ}$, respectively. In some cases, depression was assessed by a psychiatrist according to the Hamilton Depression Rating Scale (HDRS) $(15,16)$, DSM-IV criteria (17), or the Beck Depression Inventory (18).

\section{Statistical Analysis}

We used RevMan 5.3 (The Nordic Cochrane Center for The Cochrane Collaboration, Copenhagen, Denmark) to perform the meta-analysis of the RCTs according to the PRISMA (Preferred Reporting Items for Systematic Reviews and Meta-Analysis) guidelines (19). The standardized mean difference (SMD) was calculated as the effect size for continuous outcomes. The accuracy of the result was reported as a $95 \%$ confidence interval (CI). $P$ $<0.05$ was considered significant. When necessary, the means and standard deviations of pretreatment-posttreatment changes were estimated according to the reported pretreatment and posttreatment data (20). Due to possible heterogeneity between each study, the DerSimonian and Laird random-effects model was used for calculating a pooled estimate of the mean difference (21). The l-square test was performed to assess the heterogeneity among these trials.

\section{ResUlts}

\section{Study Selection and Characteristics of Included Studies}

Figure 1 illustrates a flowchart of the study selection process. We initially identified 305 potential trials but excluded 105 duplicates and 97 ineligible articles after screening their titles and abstracts. Subsequently, 103 additional reports were excluded as follows: 55 were on different topics, 14 used different comparisons, 14 were review articles, 6 were systematic reviews, 3 were protocols, 1 was a cohort study, and 1 was a case study. Finally, the remaining 9 RCTs were further analyzed.

The characteristics of these eligible studies $(14,22$ 29) are summarized in Table 1. These 9 RCTs were published between 2002 and 2018, with sample sizes of 20 to 80 patients. The mean participant age ranged from 29 to 52 years; however, an earlier trial in 2002 by Gür et al (29) did not offer any information on age. Most RCTs only included women, except that the trial by Gür et al (28) enrolled some male patients and the trial by Ruaro et al (24) enrolled one man in the placebo group. All patients had been diagnosed with fibromyalgia. For patient diagnosis, 6 RCTs $(14,22,24,27-29)$ used the American College of Rheumatology's diagnostic criteria (30), one (23) used the American Rheumatology Society's criteria, and the diagnostic criteria were not mentioned in 2 RCTs $(25,26)$. Patients continued their usual pharmacological therapy in one RCT (22), whereas 3 RCTs did not mention whether the patients were taking medication concurrently (24-26), some of the patients in one RCT continued their regular medication for fibromyalgia (14), and the remaining 4 claimed that no patients took analgesic, anti-inflammatory medications or central nervous system drugs during the study period.

Regarding laser parameters, 6 RCTs $(23,24,26-29)$ used GaAlAs or Ga-AS laser, one (25) used Girlase, one RCT employed a 9-diode cluster device containing mul- 
Studies identified through PubMed(74), Embase(188), and Cochrane(43) databases

for potentially relevant trials $(\mathrm{n}=305)$

Duplicates $(\mathrm{n}=105)$

Studies excluded after screening

titles and abstracts $(n=97)$

Potentially relevant studies retrieved for further assessment $(\mathrm{n}=103)$

Studies excluded

Different topics $(\mathrm{n}=55)$

Different comparisons $(n=14)$

Reviews $(\mathrm{n}=14)$

Systematic Reviews $(\mathrm{n}=6)$

Protocols $(n=3)$

Cohort study $(\mathrm{n}=1)$

Case study $(\mathrm{n}=1)$

Eligible studies fulfilling inclusion for systematic review $(n=9)$

Fig. 1. Flow chart of the process of study selection.

tiple light sources (LLLT and light-emitting diode [LED]) (22), and the latest RCT used a $\mathrm{DMC}^{\circledR}$ Photon Laser III device (14). Therefore, we performed a subgroup analysis to differentiate the efficacy of monowavelength LLLT vs an LLLT/LED combination. Laser wavelength ranged from 640 to $950 \mathrm{~nm}$ and power from approximately 0.9 to $1000 \mathrm{MW}$. The follow-up period of the $8 \mathrm{RCTs}$ ranged from 2 to 10 weeks; one RCT further followed for 6 months. Five studies $(24,25,27-29)$ involving 173 patients evaluated the effectiveness of LLLT by comparing with placebo laser. Three RCTs compared LLLT plus stretching exercise with stretching exercise alone $(14,23,26)$. One RCT designated patients into 4 groups: control group, phototherapy group, stretching and aerobic exercise training group, and phototherapy plus stretching and aerobic exercise training group (22).

\section{Study Quality}

As shown in Table 2, the methodological quality of 9 RCTs was assessed (14,22-29). Five RCTs $(14,22,24,28,29)$ reported acceptable methods of randomization, but none described allocation concealment methods. Eight RCTs $(14,22-25,27-29)$ reported patient blinding by applying placebo or sham laser treatment; the remaining
RCT (26) did not provide any relevant blinding information. Three RCTs by Armagan et al (27), da Silva et al (22) and Germano (14) blinded outcome assessors; da Silva et al also blinded the phototherapy programmer. Eight RCTs used an intention-to-treat analysis without loss to follow-up. However, $20 \%$ of the patients withdrew from one RCT (26) without reporting the reason, so per-protocol analysis was used for that study. One RCT reported higher variability of emitted power and energy dose of laser (26).

\section{Comparison of LLLT and Placebo LLLT}

\section{Primary Outcome (FIQ Score, Pain, and Number of Tender Points)}

The meta-analysis showed significant improvement in FIQ score after monowavelength LLLT than that after placebo laser treatment (pooled SMD: 1.16; $95 \% \mathrm{Cl}, 0.64-1.69 ; I^{2}=47 \%$; Fig. 2). The severity of pain was also significantly reduced in the monowavelength LLLT group (pooled SMD: 1.18; $95 \% \mathrm{Cl}, 0.82-1.54, \mathrm{I}^{2}=$ $0 \%$; Fig. 3) compared with the placebo groups. A significant decrease in the number of tender points after monowavelength LLLT was also noted (SMD: 1.01; 95\% 
Table 1. Characteristics of the selected RCTs.

\begin{tabular}{|c|c|c|c|c|c|}
\hline Study & $\begin{array}{c}\text { Inclusion } \\
\text { Criteria }\end{array}$ & $\begin{array}{c}\text { No. of } \\
\text { Patients }\end{array}$ & Age (yrs) & Intervention & Outcomes \\
\hline $\begin{array}{l}\text { Germano } \\
(14), 2018 \\
\text { (Brazil) }\end{array}$ & $\begin{array}{l}\text { Diagnosed with } \\
\text { FM by ACR } \\
\text { criteria }\end{array}$ & $\begin{array}{l}\text { I: } 11(0 \%) \\
\text { C: } 11(0 \%)\end{array}$ & $\begin{array}{l}\text { I: } 39.73 \pm 5.25 \\
\text { C: } 40.36 \pm 7.24\end{array}$ & $\begin{array}{l}\text { I: functional exercise program ( } 40 \text { to } 60 \mathrm{~min} / \\
\text { session) associated with active phototherapy } \\
(808 \mathrm{~nm}, 100 \mathrm{~mW} \text {, continuous, } 4 \text { J, and } 142.85 \\
\mathrm{J} / \mathrm{cm}^{2} \text { on } 17 \text { tender points immediately after } \\
\text { exercise, } 40 \mathrm{~s} / \text { site } 3 \text { times } / \mathrm{wk}) \times 8 \text { wks } \\
\text { C: functional exercise program ( } 40 \text { to } \\
60 \mathrm{~min} / \mathrm{session}) \text { associated with placebo } \\
\text { phototherapy ( } 3 \text { times } / \mathrm{wk}) \times 8 \text { wks }\end{array}$ & $\begin{array}{l}\text { FIQ, VAS, no. of tender } \\
\text { points, Beck Depression } \\
\text { Inventory, pain } \\
\text { threshold, functional } \\
\text { performance, muscle } \\
\text { performance (flexibility, } \\
\text { strength) }\end{array}$ \\
\hline $\begin{array}{l}\text { da Silva (22), } \\
2018 \text { (Brazil) }\end{array}$ & $\begin{array}{l}\text { Diagnosed as } \\
\text { FM by ACR } \\
\text { criteria on FIQ } \\
\text { for }>5 \text { yrs, } \geq 35 \\
\text { y/o women }\end{array}$ & $\begin{array}{l}\text { I: } 20\left(0 \%^{\mathrm{a}}\right) \\
\text { C1: } 20(0 \%) \\
\text { C2: } 20(0 \%) \\
\text { C3: } 20(0 \%)\end{array}$ & $\begin{array}{l}\text { Overall: } \\
40 \pm 2\end{array}$ & $\begin{array}{l}\text { I: Phototherapy (a cluster with } 9 \text { diodes-1 } \\
\text { super-pulsed infrared } 905 \mathrm{~nm}, 4 \text { LED of } 640 \\
\mathrm{~nm}, 4 \text { LED of } 875 \mathrm{~nm}, 39.3 \mathrm{~J} \& 5 \mathrm{~min} / \text { point } \mathrm{x} \\
10 \text { sites) } \text { x } 10 \mathrm{wks} \\
\text { C1: Placebo phototherapy x } 10 \mathrm{wks} \\
\text { C2: Phototherapy + exercise }{ }^{\mathrm{b}} \times 10 \mathrm{wks} \\
\text { C3: Placebo phototherapy + } \text { exercise }^{\mathrm{b}} \times 10 \mathrm{wks}\end{array}$ & $\begin{array}{l}\text { FIQ, VAS, fatigue, body } \\
\text { stiffness, no. of tender } \\
\text { points, depression, } \\
\text { anxiety, SF-36; } \\
(10 \text { wks })^{\mathrm{e}}\end{array}$ \\
\hline $\begin{array}{l}\text { Vayvay } \\
(23), 2016 \\
\text { (Turkey) }\end{array}$ & $\begin{array}{l}\text { Diagnosed with } \\
\text { FM by ARS } \\
\text { criteria, } \geq 18 \\
\text { y/o, continuous } \\
\text { chronic pain } \geq \\
6 \text { mos }\end{array}$ & $\begin{array}{l}\text { I: } 15(0 \%) \\
\text { C1: } 15(0 \%) \\
\text { C2: } 15(0 \%)^{c}\end{array}$ & $\begin{array}{l}\text { I: } 36.4 \pm 8.3 \\
\text { C1: } 38 \pm 8.4 \\
\text { C2: } 38 \pm 9.9^{c}\end{array}$ & $\begin{array}{l}\text { I: Ga-AS Laser }\left(850 \mathrm{~nm} ; 40 \mathrm{~mW} ; 2 \mathrm{~J} / \mathrm{cm}^{2} ; 50-\right. \\
60 \mathrm{~Hz}, 3 \mathrm{~min} / \text { painful point on back and head) } \\
+ \text { exercise } \mathrm{x} 3 \mathrm{wks} \\
\mathrm{C} 1 \text { : Placebo laser }+ \text { exercise } \mathrm{d} \text { } 3 \text { wks } \\
\mathrm{C} 2 \text { : Kinesiotape on the back for } 3 \text { wks }+ \\
\text { exercise }^{\mathrm{d}} \times 3 \text { wks }\end{array}$ & $\begin{array}{l}\text { FIQ, VAS, SF-36, Beck } \\
\text { Depression Inventory } \\
\text { Anxiety Level (3 wks) }\end{array}$ \\
\hline $\begin{array}{l}\text { Ruaro (24), } \\
2014 \text { (Brazil) }\end{array}$ & $\begin{array}{l}\text { Diagnosed with } \\
\text { FM by ACR } \\
\text { criteria }\end{array}$ & $\begin{array}{l}\text { I: } 10(0 \%) \\
\text { C: } 10(10 \%)\end{array}$ & $\begin{array}{l}\text { I: } 39.4(34-45) \\
\text { C: } 43.4(33-55)\end{array}$ & $\begin{array}{l}\text { I: GaAlAs laser }\left(670 \mathrm{~nm}, 20 \mathrm{~mW}, 4 \mathrm{~J} / \mathrm{cm}^{2} \text { on }\right. \\
18 \text { tender points, } 3 \text { times } / \mathrm{wk}) \mathrm{x} 4 \mathrm{wks} \\
\text { C: Placebo laser } \mathrm{x} 4 \mathrm{wks}\end{array}$ & $\begin{array}{l}\text { FIQ, McGill Pain } \\
\text { Questionnaire, VAS ( } 4 \\
\text { wks) }\end{array}$ \\
\hline $\begin{array}{l}\text { Fernández } \\
(25), 2011 \\
\text { (Spain) }\end{array}$ & $\begin{array}{l}\text { Diagnosis of FM } \\
\text { for } 3-10 \text { yrs, } 36- \\
61 \text { y/o woman }\end{array}$ & $\begin{array}{l}\text { I: } 16(0 \%) \\
\text { C: } 15(0 \%)\end{array}$ & $\begin{array}{l}\text { I: } 51.6 \pm 6.18 \\
\text { C: } 52.4 \pm 5.88\end{array}$ & $\begin{array}{l}\text { I: Girlase } \mathrm{E} 1.1010(905+10 \mathrm{~nm}, 0.70 \mathrm{~mJ} / \\
\text { drive, } 1000 \mathrm{~mW} \text { boost of the drives, pulsed, } 1 \\
\text { min/frequency x } 6 \text { on } 7 \text { points }) \text { x } 8 \text { wks } \\
\text { C: Placebo laser } \mathrm{x} 8 \text { wks }\end{array}$ & $\begin{array}{l}\text { CRD (FIQ), Generalized } \\
\text { pain, fatigue ( } 8 \text { wks) }\end{array}$ \\
\hline $\begin{array}{l}\text { Matsutani } \\
(26), 2007 \\
\text { (Brazil) }\end{array}$ & $\begin{array}{l}\text { Diagnosed as } \\
\text { FM for } 25-60 \\
\text { y/o, exclude } \\
\text { neoplasia }\end{array}$ & $\begin{array}{l}\text { I: } 10(0 \%) \\
\text { C: } 10(0 \%)\end{array}$ & $\begin{array}{l}\text { I: } 44 \text { (28-60) } \\
\text { C: } 45 \text { (31-57) }\end{array}$ & $\begin{array}{l}\text { I: GaAlAs laser }\left(830 \mathrm{~nm}, 3 \mathrm{~J} / \mathrm{m}^{2} \text {, average } 30\right. \\
\mathrm{mW} \text {, continuous })+ \text { exercise }^{\mathrm{d}} 1 \mathrm{~h} \text { BIW x } 5 \text { wks } \\
\text { C: Placebo laser }+ \text { exercise }^{\mathrm{d}} 1 \mathrm{~h} \text { BIW x } 5 \text { wks }\end{array}$ & FIQ, VAS, SF-36 (5 wks) \\
\hline $\begin{array}{l}\text { Amargan } \\
(27), 2006 \\
\text { (Turkey) }\end{array}$ & $\begin{array}{l}\text { Diagnosed with } \\
\text { FM by ACR } \\
\text { criteria }\end{array}$ & $\begin{array}{l}\text { I: } 16(10 \%) \\
\text { C: } 16(0 \%)\end{array}$ & $\begin{array}{l}\text { I: } 38.9 \pm 4.9 \\
\text { C: } 37.6 \pm 5.9\end{array}$ & $\begin{array}{l}\text { I: GaAlAs laser }(830 \mathrm{~nm}, 50 \mathrm{~mW} \text {, continuous, } \\
1 \mathrm{~min} \& 2 \mathrm{~J} / \mathrm{tender} \text { point), } 5 \text { days/wk x } 2 \mathrm{wks} \\
\text { C: Placebo laser } 5 \text { days/wk x } 2 \text { wks }\end{array}$ & $\begin{array}{l}\text { FIQ, no. of tender } \\
\text { points, morning } \\
\text { stiffness, VSGI } \\
(2 \mathrm{wks})\end{array}$ \\
\hline $\begin{array}{l}\text { Gür (28), } \\
2002 \\
\text { (Turkey) }\end{array}$ & $\begin{array}{l}\text { Diagnosed with } \\
\text { FM by ACR } \\
\text { criteria; exclude } \\
\text { major clinical } \\
\text { conditions other } \\
\text { than FM }\end{array}$ & $\begin{array}{l}\text { I: } 25(20 \%) \\
\text { C1: } 25(24 \%) \\
\text { C2: } 25(16 \%)^{c}\end{array}$ & $\begin{array}{l}\text { I: } 30.4 \pm 6.9 \\
\text { C1: } 28.5 \pm 6.3 \\
\text { C2: } 30.1 \pm 8.7^{c}\end{array}$ & $\begin{array}{l}\text { I: Ga-As laser (904 } \mathrm{nm} \text {, average } 11.2 \mathrm{~mW} \text {, } \\
\left.2 \mathrm{~J} / \mathrm{cm}^{2}, 2.8 \mathrm{kHz}\right) 3 \mathrm{~min} / \text { tender point every } \\
\text { afternoon x } 2 \mathrm{wks} \text { (except weekend) } \\
\mathrm{C} 1 \text { : Placebo laser x } 2 \mathrm{wks} \\
\mathrm{C} 2 \text { : Amitriptyline } 10 \mathrm{mg} \text { at bedtime } \mathrm{x} 8 \mathrm{wks}^{\mathrm{c}}\end{array}$ & $\begin{array}{l}\text { FIQ, depression (HDRS, } \\
\text { DSM-IV), pain, no. } \\
\text { of tender points, } \\
\text { morning stiffness, sleep } \\
\text { disturbance, fatigue ( } 2 \\
\text { wks/6 mos) }\end{array}$ \\
\hline $\begin{array}{l}\text { Gür (29), } \\
2002 \text { (earlier } \\
\text { published) } \\
\text { (Turkey) }\end{array}$ & $\begin{array}{l}\text { Diagnosed with } \\
\text { FM by ACR } \\
\text { criteria; exclude } \\
\text { major clinical } \\
\text { conditions other } \\
\text { than FM }\end{array}$ & $\begin{array}{l}\text { I: } 20(0 \%) \\
\text { C: } 20(0 \%)\end{array}$ & Not mentioned & $\begin{array}{l}\text { I: Ga-As laser }(904 \mathrm{~nm} \text {, average } 11.2 \mathrm{~mW} \text {, } \\
\left.2 \mathrm{~J} / \mathrm{cm}^{2}, 2.8 \mathrm{kHz}\right) 3 \mathrm{~min} / \text { tender point every } \\
\text { afternoon x } 2 \text { wks (except weekends) } \\
\text { C: Placebo laser x } 2 \text { wks }\end{array}$ & $\begin{array}{l}\text { Pain, morning stiffness, } \\
\text { no. of tender points, } \\
\text { sleep disturbance, } \\
\text { fatigue ( } 2 \mathrm{wks})\end{array}$ \\
\hline
\end{tabular}

Abbreviations: ACR, American College of Rheumatology; ARS, American Rheumatology Society; C, Control group; CRD, Cuaderno de recogida de datos; FIQ, Fibromyalgia Impact Questionnaire; FM, Fibromyalgia; HDRS, Hamilton Depression Rating Scale; I, Intervention group; LED, light-emitting diode; SF-36, 36-item Short-Form Health Survey; VAS, Visual Analog Scale of pain; VSGI, global improvement as reported on a verbal scale. Age was presented as mean \pm SD or mean (range). ${ }^{a}(): \%$ men; ${ }^{b}$ stretching and aerobic exercise; ${ }^{c}$ not included for our analysis; ${ }^{\mathrm{d}}$ stretching; ${ }^{\mathrm{e}}()$ : duration of outcome follow-up 
Table 2. Methodological quality assessment of the selected RCTs.

\begin{tabular}{|c|c|c|c|c|c|c|c|}
\hline Study & $\begin{array}{l}\text { Allocation } \\
\text { Generation }\end{array}$ & $\begin{array}{l}\text { Allocation } \\
\text { Concealment }\end{array}$ & Blinding & $\begin{array}{l}\text { Data } \\
\text { Analysis }\end{array}$ & \begin{tabular}{|l} 
Loss to \\
Follow-up
\end{tabular} & $\begin{array}{l}\text { Patient } \\
\text { Gender }\end{array}$ & Other Relevant Remarks \\
\hline $\begin{array}{l}\text { Germano } \\
(14), 2018\end{array}$ & Adequate & Unclear & $\begin{array}{l}\text { Patients, } \\
\text { evaluators }\end{array}$ & ITT & $0 \%$ & Only women & $\begin{array}{l}\text { Some patients continued regular } \\
\text { medications for fibromyalgia in } \\
2 \text { groups }\end{array}$ \\
\hline $\begin{array}{l}\text { da Silva (22), } \\
2018\end{array}$ & Adequate & Unclear & $\begin{array}{l}\text { Patients, } \\
\text { phototherapy } \\
\text { programmer, } \\
\text { and outcome } \\
\text { assessor }\end{array}$ & ITT & $0 \%$ & Only women & $\begin{array}{l}\text { Age distribution not reported; } \\
\text { unknown pharmacological } \\
\text { therapy for fibromyalgia in } 2 \\
\text { groups }\end{array}$ \\
\hline $\begin{array}{l}\text { Vayvay (23), } \\
2016\end{array}$ & Unclear & Unclear & Patients & ITT & $0 \%$ & Only women & - \\
\hline $\begin{array}{l}\text { Ruaro (24), } \\
2014\end{array}$ & Adequate & Unclear & Patients & ITT & $0 \%$ & $\begin{array}{l}\text { Only women, } \\
\text { with one man } \\
\text { in the placebo } \\
\text { group }\end{array}$ & $\begin{array}{l}\text { Unknown pharmacological } \\
\text { therapy for fibromyalgia in } 2 \\
\text { groups }\end{array}$ \\
\hline $\begin{array}{l}\text { Fernández } \\
\text { (25), } 2011\end{array}$ & Unclear & Unclear & Patients & ITT & $0 \%$ & Only women & $\begin{array}{l}\text { Unknown pharmacological } \\
\text { therapy for fibromyalgia in } 2 \\
\text { groups }\end{array}$ \\
\hline $\begin{array}{l}\text { Matsutani } \\
\text { (26), } 2007\end{array}$ & Unclear & Unclear & Unclear & PP & $20 \%$ & Only women & $\begin{array}{l}\text { Unclear reasons for, and } \\
\text { unknown distribution of, loss } \\
\text { of follow-up; may have high } \\
\text { variability of emitted power } \\
\text { and energy dose; unknown } \\
\text { pharmacological therapy for } \\
\text { fibromyalgia in } 2 \text { groups } \\
\end{array}$ \\
\hline $\begin{array}{l}\text { Armagan } \\
(27), 2006\end{array}$ & Unclear & Unclear & $\begin{array}{l}\text { Patients and } \\
\text { evaluators }\end{array}$ & ITT & $0 \%$ & Only women & - \\
\hline $\begin{array}{l}\text { Gür (28), } \\
2002\end{array}$ & Adequate & Unclear & Patients & ITT & $0 \%$ & - & - \\
\hline $\begin{array}{l}\text { Gür (29), } \\
2002 \text { (earlier } \\
\text { published) }\end{array}$ & Adequate & Unclear & Patients & ITT & $0 \%$ & Only women & Age distribution not reported \\
\hline
\end{tabular}

Abbreviations: ITT, intention to treat; PP, per-protocol; RCT, randomized controlled trial.

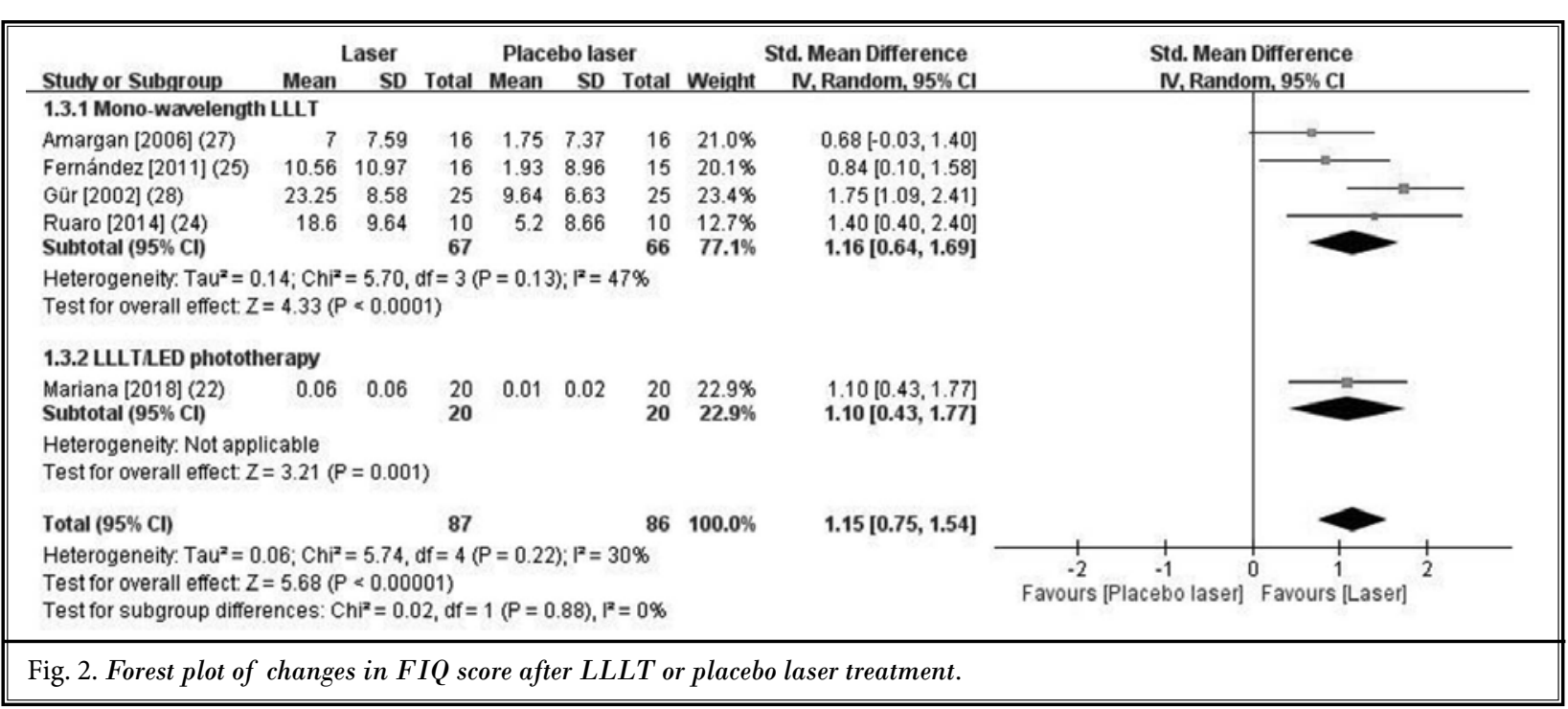


$\mathrm{Cl}, 0.49-1.52 ; I^{2}=49 \%$; Fig. 4). As for the combined LLLT/ LED phototherapy, the only RCT evaluating efficacy showed significant improvement in FIQ, pain, and number of tender points compared with the placebo group. The effect of combined LLLT/LED phototherapy on pain relief and reduction in the number of tender points seemed to be more obvious than monowavelength LLLT (Figs. 3 and 4).

\section{Secondary Outcomes (Fatigue, Stiffness, Depression, and Anxiety)}

In the monowavelength LLLT group, our analysis showed significant improvements in the severity of fatigue (pooled SMD: $1.4 ; 95 \% \mathrm{Cl}, 0.96-1.8$ ), stiffness (pooled SMD: 0.92, 95\% Cl: 0.36-1.48), depression (pooled SMD: $1.46,95 \% \mathrm{Cl}: 0.93-2.00$ ), and anxiety (pooled SMD: 1.46, 95\% Cl, 0.45-2.47). On the other hand, the only one RCT evaluating the efficacy of the combined LLLT/LED phototherapy demonstrated significant improvement in the severity of fatigue, stiffness, and anxiety, but not depression, when compared with those in the placebo laser group.

\section{Comparison of LLLT Plus Exercise and Placebo Laser Treatment Plus Exercise}

Three RCTs evaluated the efficacy of monowavelength LLLT with exercise $(14,23,26)$. There was no significant difference between the monowavelength

\begin{tabular}{|c|c|c|c|c|c|c|c|c|c|c|c|}
\hline \multirow{3}{*}{$\begin{array}{l}\text { Study or Subgroup } \\
\text { 1.4.1 Mono-wavelength LLI }\end{array}$} & \multicolumn{2}{|c|}{\begin{tabular}{rr}
\multicolumn{2}{c}{ Laser } \\
Mean SD
\end{tabular}} & \multicolumn{4}{|c|}{ Placebo laser } & \multirow{2}{*}{\multicolumn{2}{|c|}{$\begin{array}{cc} & \text { Std. Mean Difference } \\
\text { Weight } & \text { N. Random, } 95 \% ~ C l \\
\end{array}$}} & \multirow{2}{*}{\multicolumn{3}{|c|}{$\begin{array}{l}\text { Std. Mean Difference } \\
\text { IV. Random. } 95 \% \mathrm{Cl}\end{array}$}} \\
\hline & Mean & SD & Iotal & Mean & SD & Iotal & & & & & \\
\hline & & & & & & & & & & & \\
\hline Fernández [2011] (25) * & 2.31 & 2.1 & 16 & 0.73 & 1.82 & 15 & $21.3 \%$ & $0.78[0.05,1.52]$ & & $=$ & \\
\hline Gür [2002] (28) \# & 1.8 & 0.51 & 25 & 1 & 0.63 & 25 & $21.7 \%$ & $1.37[0.75,2.00]$ & & $\rightarrow$ & \\
\hline Gûr [2002] (earlier published) (29) \# & 1.82 & 0.54 & 20 & 1.04 & 0.71 & 20 & $21.5 \%$ & $1.21[0.53,1.89]$ & & $\rightarrow$ & \\
\hline $\begin{array}{l}\text { Ruaro [2014] (24)\$ } \\
\text { Subtotal }(95 \% \mathrm{Cl})\end{array}$ & 2.7 & 1.14 & $\begin{array}{l}10 \\
71\end{array}$ & 1.1 & 1.16 & $\begin{array}{l}10 \\
70\end{array}$ & $\begin{array}{l}20.2 \% \\
84.7 \%\end{array}$ & $\begin{array}{l}1.33[0.34,2.32] \\
1.18[0.82,1.54]\end{array}$ & & $\rightarrow$ & \\
\hline \multicolumn{12}{|c|}{$\begin{array}{l}\text { Heterogeneity. Tau }=0.00 ; \mathrm{Chi}^{2}=1.61, \mathrm{df}=3(P=0.66) ; \mathrm{I}^{2}=0 \% \\
\text { Test for overall effect: } Z=6.38(P<0.00001)\end{array}$} \\
\hline \multicolumn{12}{|l|}{ 1.4.2 LLLT/LED phototherapy } \\
\hline $\begin{array}{l}\text { Mariana }[2018](22)^{2} \\
\text { Subtotal }(95 \% \mathrm{CI})\end{array}$ & 0.62 & 0.08 & $\begin{array}{l}20 \\
20\end{array}$ & 0.14 & 0.03 & $\begin{array}{l}20 \\
20\end{array}$ & $\begin{array}{l}15.3 \% \\
15.3 \%\end{array}$ & $\begin{array}{l}7.79[5.89,9.69] \\
7.79[5.89,9.69]\end{array}$ & & & \\
\hline \multicolumn{12}{|c|}{$\begin{array}{l}\text { Heterogeneity. Not applicable } \\
\text { Test for overall effect: } Z=8.03(P<0.00001)\end{array}$} \\
\hline Total $(95 \% \mathrm{Cl})$ & & & 91 & & & 90 & $100.0 \%$ & $2.18[0.90,3.47]$ & & & \\
\hline \multicolumn{9}{|c|}{$\begin{array}{l}\text { Heterogeneity. Tauz }=1.88 ; \mathrm{Chi}^{2}=46.40, \mathrm{df}=4(\mathrm{P}<0.00001) ; \mathrm{I}^{2}=91 \% \\
\text { Test for overall effect: } Z=3.33(P=0.0009) \\
\text { Test for subgroup differences: } C h \mathrm{I}^{2}=44.80, \mathrm{df}=1(P<0.00001), \mathrm{I}^{2}=97.8 \%\end{array}$} & 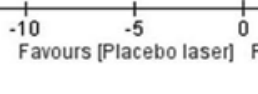 & $\begin{array}{c}1 \\
5 \\
\text { Favours [Laser] }\end{array}$ & 10 \\
\hline$*: 1-10 ; \#: 0-4 ; \$: 0-10 ;{ }^{a}:$ VAS, 10 & rule & & & & & & & & & & \\
\hline
\end{tabular}

Fig. 3. Forest plot of changes in pain severity after LLLT or placebo laser treatment.

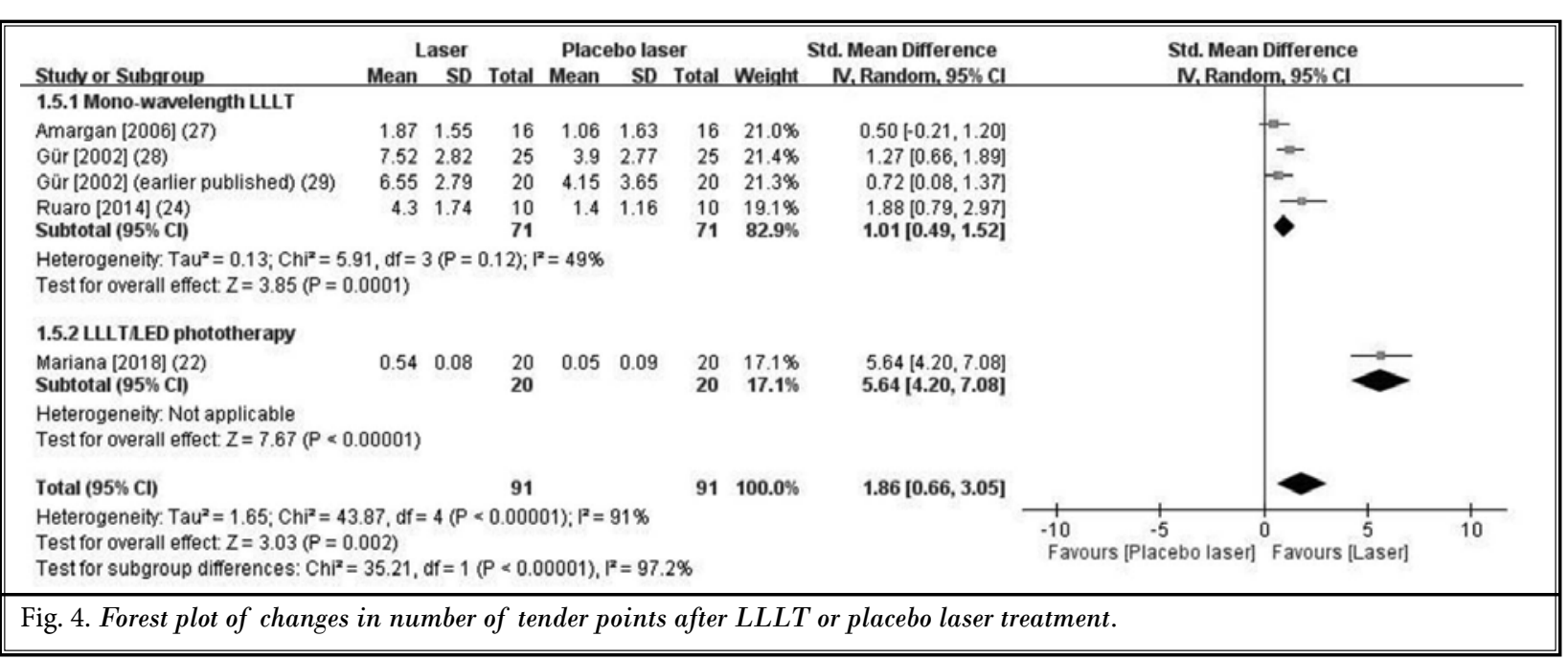


Pain Physician: May/June 2019: 22:241-254

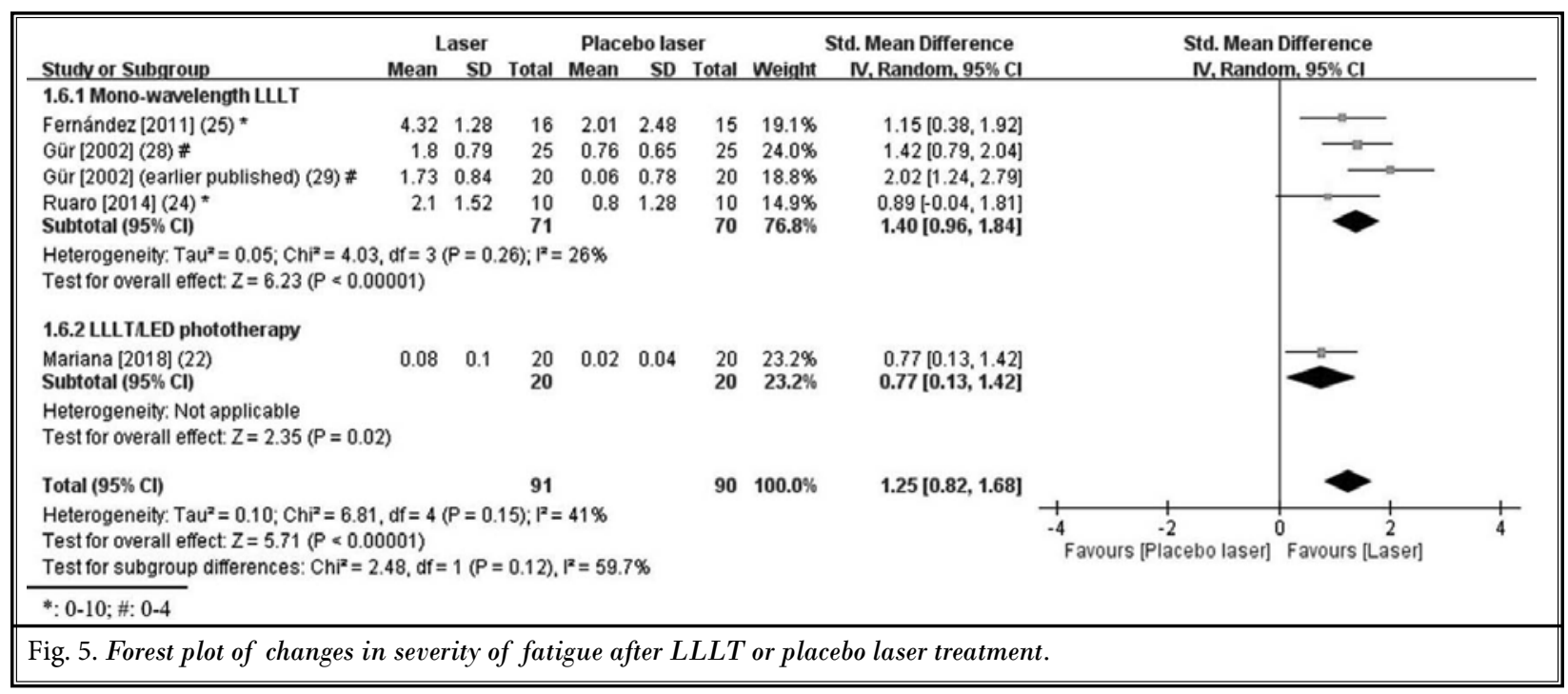

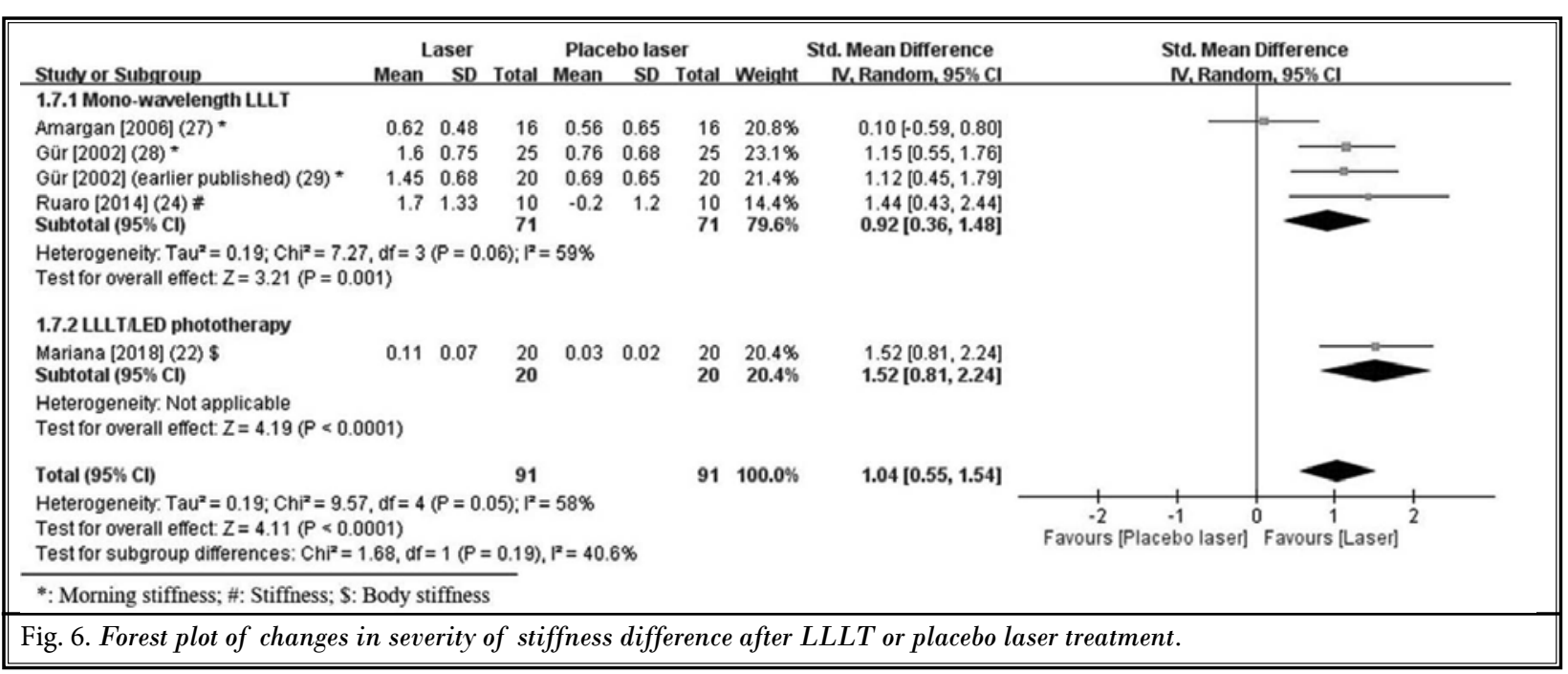

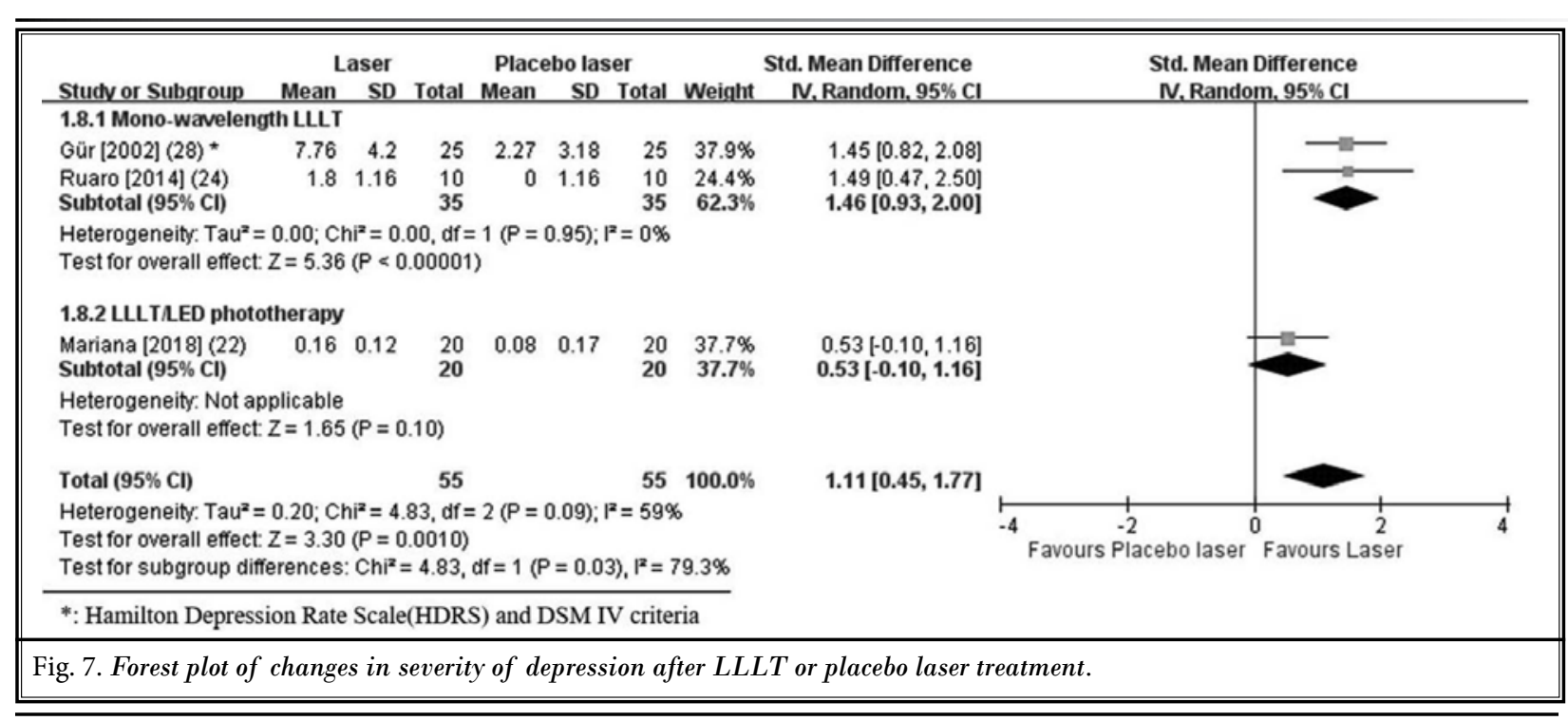




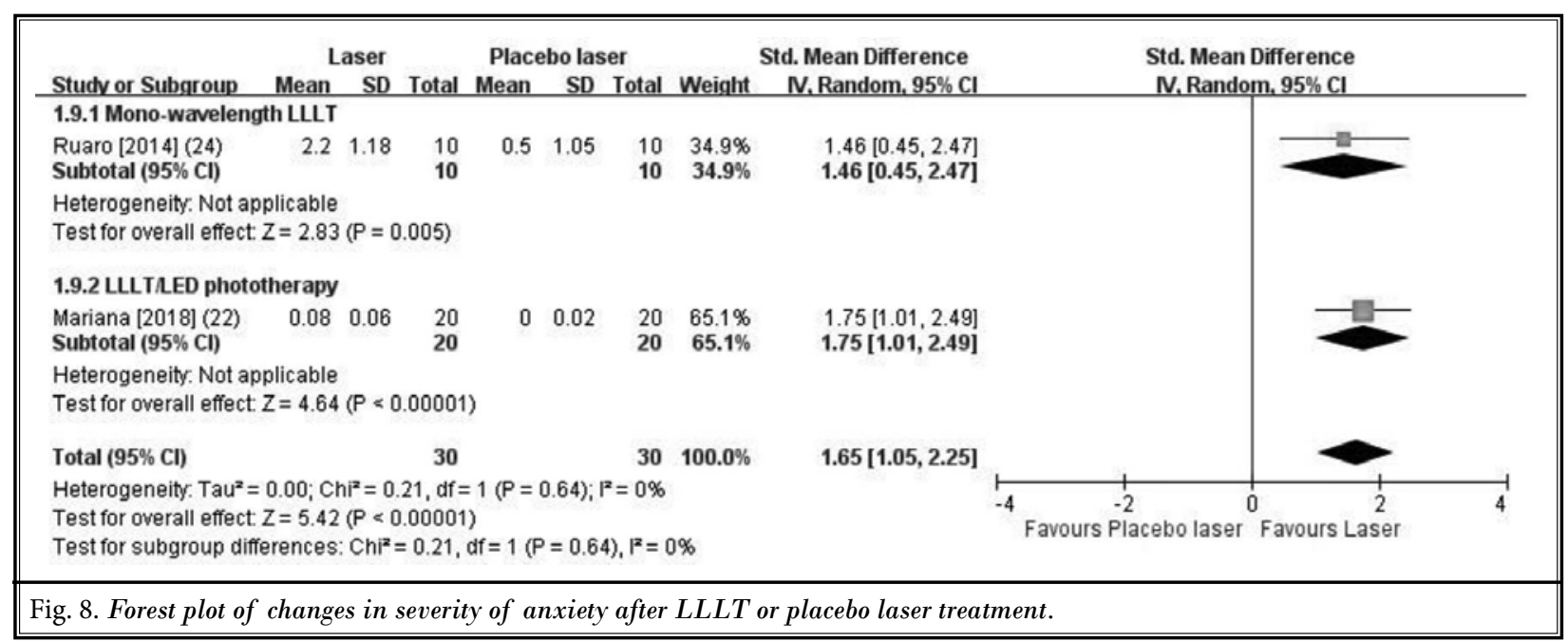

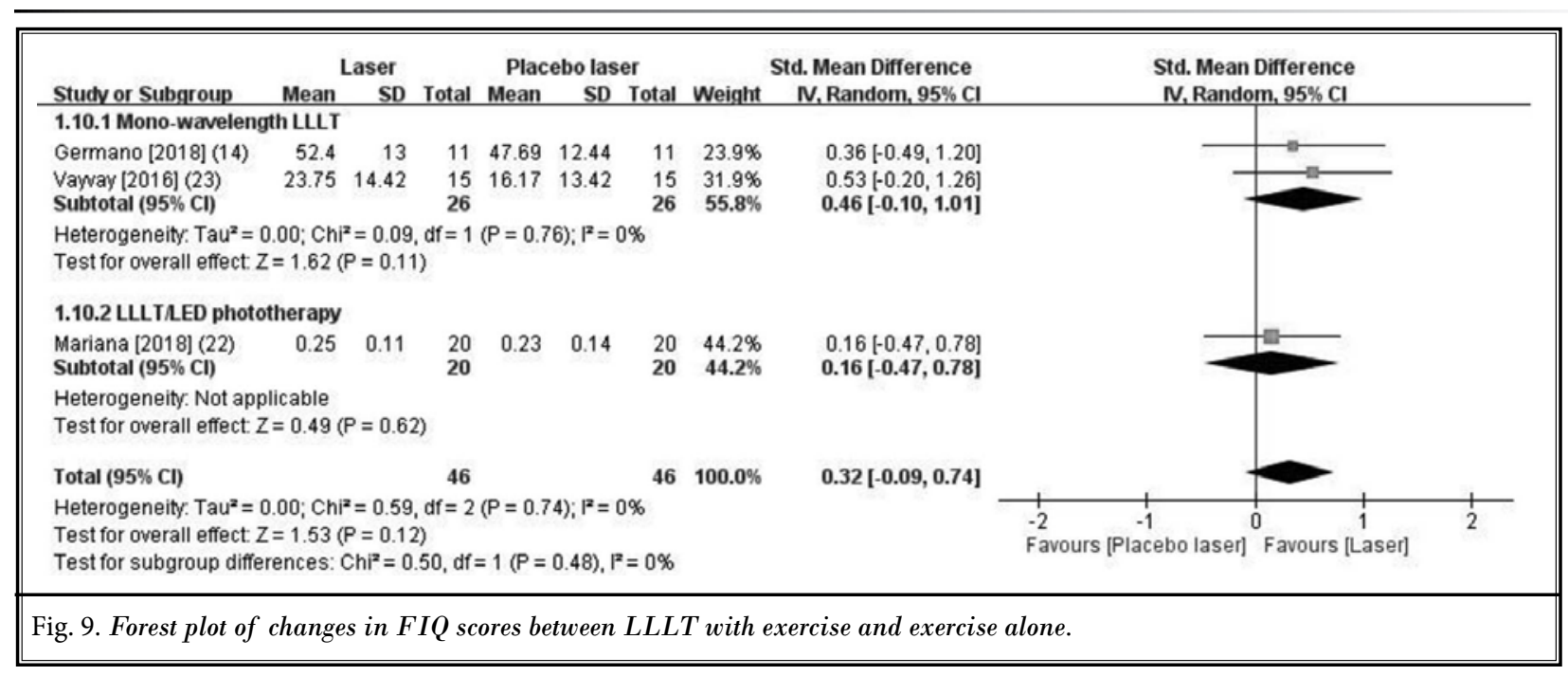

LLLT plus standardized exercise program and exercise program alone in the primary outcomes of FIQ score (pooled SMD: $0.34 ; 95 \% \mathrm{Cl},-0.17$ to $0.85 ; \mathrm{I}^{2}=14 \%$; Fig. 9), pain (pooled SMD: $0.46 ; 95 \% \mathrm{Cl},-0.10$ to $1.01 ; \mathrm{I}^{2}=$ $0 \%$; Fig. 10) and number of tender points (pooled SMD: $0.59 ; 95 \% \mathrm{Cl},-0.26$ to $1.45 ;$ Fig. 11 ) and secondary outcomes of fatigue, stiffness, anxiety, or depression (SMD: $-0.16 ; 95 \% \mathrm{Cl},-1.04$ to 0.72 ; SMD: $0.08 ; 95 \% \mathrm{Cl},-0.79$ to 0.96; SMD: $0.09 ; 95 \% \mathrm{Cl},-0.80$ to $0.98 ; 1^{2}=70 \%$; SMD: $-0.38 ; 95 \% \mathrm{Cl}, 1.27-0.50$, respectively; Figs. 12-15).

Compared with standardized exercise alone, LLLT/ LED combination phototherapy plus exercise program, as reported in only one RCT, provided significant additional benefit in relieving the primary outcome of the severity of pain and number of tender points (SMD: 5.20; 95\% Cl, 3.85-6.55 and SMD: 7.02; 95\% Cl,
5.29-8.76, respectively) and the secondary outcome of fatigue (SMD: $1.35 ; 95 \% \mathrm{Cl}, 0.65-2.04$ ), but it not decrease in FIQ score, severity of stiffness, or psychiatric symptoms (Figs. 9-15) (22).

\section{Side Effects}

Four RCTs $(22,24,27,29)$ reported no side effects of LLLT in patients with fibromyalgia, consistent with previous studies $(6,10,31)$. However, the remaining RCTs did not report on side effects $(14,23,25,26,28)$.

\section{Discussion}

LLLT has been introduced as a noninvasive, therapeutic intervention for pain in several musculoskeletal disorders. Some mechanisms, such as increased nociceptive threshold, endorphin production, and downstream 


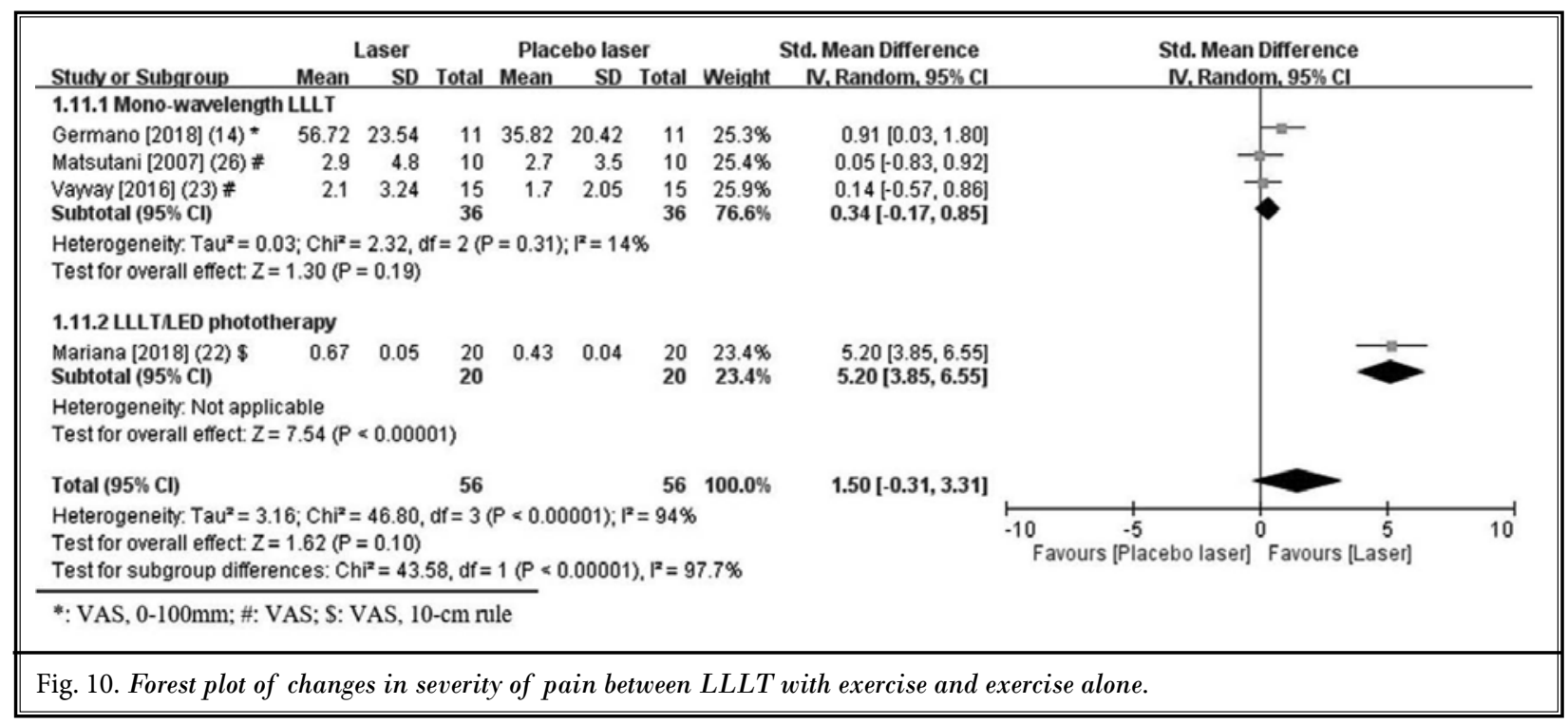

\begin{tabular}{|c|c|c|c|c|c|c|c|c|c|c|c|}
\hline \multirow[b]{2}{*}{ Studv or Subgroup } & \multicolumn{2}{|c|}{ Laser } & \multicolumn{4}{|c|}{ Placebo laser } & \multirow{2}{*}{\multicolumn{2}{|c|}{$\begin{array}{cc} & \text { Std. Mean Difference } \\
\text { Weight } & \text { N. Random, 95\% Cl }\end{array}$}} & \multirow{2}{*}{\multicolumn{3}{|c|}{$\begin{array}{l}\text { Std. Mean Difference } \\
\text { IV. Random, } 95 \% \mathrm{Cl}\end{array}$}} \\
\hline & Mean & & Total & Mean & SD & Iotal & & & & & \\
\hline \multicolumn{12}{|c|}{ 1.12.1 Mono-wavelength LLLT } \\
\hline $\begin{array}{l}\text { Germano [2018] (14) } \\
\text { Subtotal (95\% Cl) }\end{array}$ & 1.82 & 1.63 & $\begin{array}{l}11 \\
11\end{array}$ & 0.64 & 2.16 & $\begin{array}{l}11 \\
11\end{array}$ & $\begin{array}{l}50.7 \% \\
\mathbf{5 0 . 7 \%}\end{array}$ & $\begin{array}{c}0.59[-0.26,1.45] \\
\mathbf{0 . 5 9}[-\mathbf{0 . 2 6}, \mathbf{1 . 4 5}]\end{array}$ & & & \\
\hline \multicolumn{12}{|c|}{$\begin{array}{l}\text { Heterogeneity: Not applicable } \\
\text { Test for overall effect: } Z=1.36(P=0.18)\end{array}$} \\
\hline \multicolumn{12}{|c|}{ 1.12.2 LLILLE phototherapy } \\
\hline $\begin{array}{l}\text { Mariana [2018] (22) } \\
\text { Subtotal (95\% Cl) }\end{array}$ & 0.85 & 0.08 & $\begin{array}{l}20 \\
20\end{array}$ & 0.24 & 0.09 & $\begin{array}{l}20 \\
20\end{array}$ & $\begin{array}{l}49.3 \% \\
\mathbf{4 9 . 3} \%\end{array}$ & $\begin{array}{l}7.02[5.29,8.76] \\
7.02[5.29,8.76]\end{array}$ & & & \\
\hline \multicolumn{12}{|c|}{$\begin{array}{l}\text { Heterogeneity. Not applicable } \\
\text { Test for overall effect: } Z=7.93 \text { ( } P<0.00001)\end{array}$} \\
\hline Total $(95 \% \mathrm{Cl})$ & & & 31 & & & 31 & $100.0 \%$ & $3.76[-2.54,10.06]$ & & & \\
\hline \multicolumn{9}{|c|}{$\begin{array}{l}\text { Heterogeneity. } \mathrm{Tau}^{2}=20.18 ; \mathrm{Ch}^{2}=42.38, \mathrm{df}=1(\mathrm{P}<0.00001) ; \mathrm{I}^{2}=98 \% \\
\text { Test for overall effect: } Z=1.17(P=0.24) \\
\text { Test for subgroup differences: } \mathrm{Ch} \mathrm{I}^{2}=42.38, \mathrm{df}=1(P<0.00001), \mathrm{I}^{2}=97.6 \%\end{array}$} & $\begin{array}{lcc}-10 & -5 & 0 \\
\text { Favours [Placebo laser] } & F\end{array}$ & $\begin{array}{c}5 \\
\text { Favours [Laser] }\end{array}$ & 10 \\
\hline
\end{tabular}

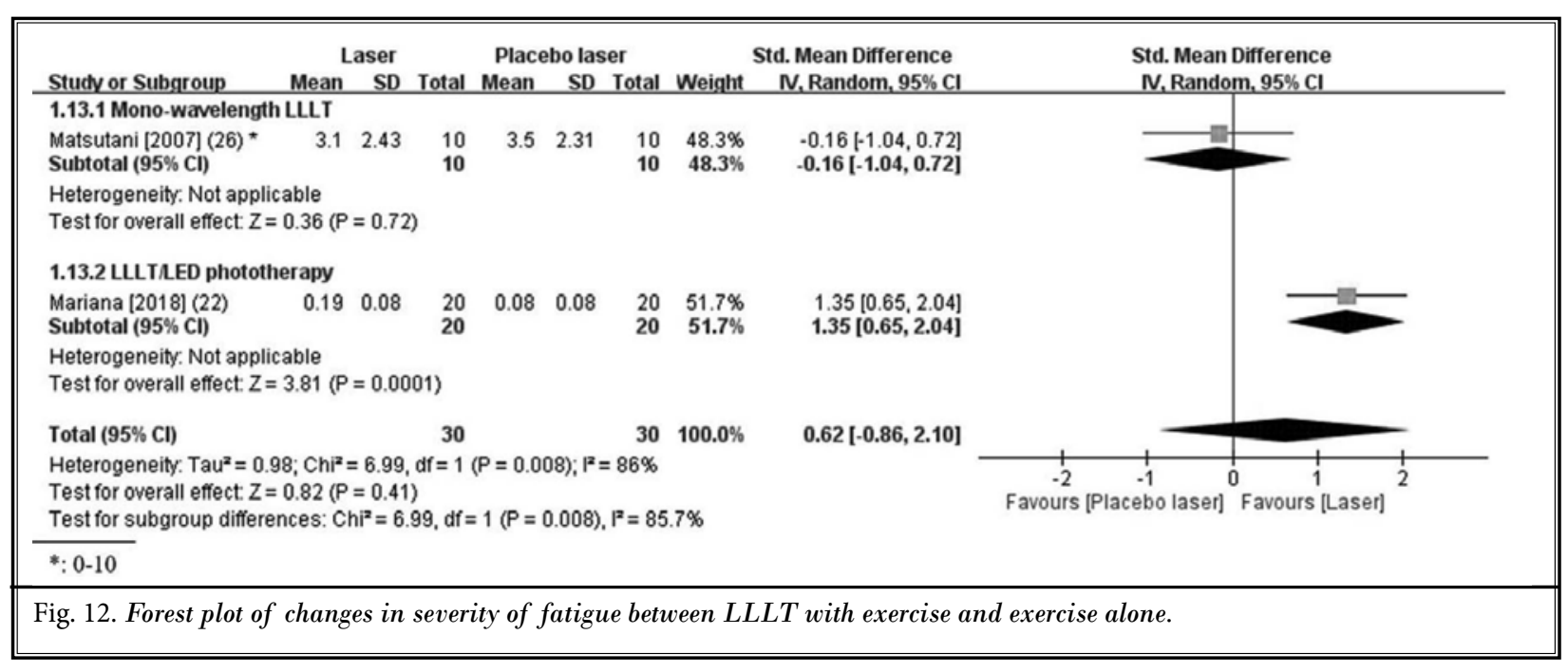


Low-Level Laser Therapy for Fibromyalgia: A Systematic Review and Meta-Analysis

\begin{tabular}{|c|c|c|c|c|c|c|c|c|c|c|c|}
\hline Stucty or Subgroup & \multicolumn{2}{|c|}{ Laser } & \multicolumn{4}{|c|}{ Placebo laser } & Weight & $\begin{array}{l}\text { Std. Mean Difference } \\
\quad \text { N. Random, } 95 \% \mathrm{Cl}\end{array}$ & \multicolumn{3}{|c|}{$\begin{array}{l}\text { Std. Mean Difference } \\
\text { N. Random. } 95 \% \mathrm{CI}\end{array}$} \\
\hline \multicolumn{12}{|c|}{ 1.14.1 Mono-wavelength LLLT } \\
\hline $\begin{array}{l}\text { Matsutani [2007] (26) * } \\
\text { Subtotal }(95 \% \mathrm{Cl})\end{array}$ & 1.1 & 2.07 & $\begin{array}{l}10 \\
10\end{array}$ & 0.9 & 2.46 & $\begin{array}{l}10 \\
10\end{array}$ & $\begin{array}{l}34.0 \% \\
34.0 \%\end{array}$ & $\begin{array}{c}0.08[-0.79,0.96] \\
0.08[-0.79,0.96]\end{array}$ & & & \\
\hline \multicolumn{12}{|c|}{$\begin{array}{l}\text { Heterogeneity. Not applicable } \\
\text { Test for overall effect: } Z=0.19(P=0.85)\end{array}$} \\
\hline \multicolumn{12}{|c|}{ 1.14.2 LLLT/LED phototherapy } \\
\hline $\begin{array}{l}\text { Mariana [2018] (22) \# } \\
\text { Subtotal (95\% Cl) }\end{array}$ & 0.1 & 0.08 & $\begin{array}{l}20 \\
20\end{array}$ & 0.06 & 0.09 & $\begin{array}{l}20 \\
20\end{array}$ & $\begin{array}{l}66.0 \% \\
66.0 \%\end{array}$ & $\begin{array}{c}0.46[-0.17,1.09] \\
0.46[-0.17,1.09]\end{array}$ & & & \\
\hline \multicolumn{12}{|c|}{$\begin{array}{l}\text { Heterogeneity. Not applicable } \\
\text { Test for overall effect: } Z=1.44(P=0.15)\end{array}$} \\
\hline \multirow{2}{*}{\multicolumn{9}{|c|}{$\begin{array}{l}\text { Heterogeneity. Tau }=0.00 ; \mathrm{Chi}^{2}=0.47, \mathrm{df}=1(P=0.49) ; \mathrm{I}^{2}=0 \% \\
\text { Test for overall effect: } Z=1.28(P=0.20) \\
\text { Test for subgroup differences: } \mathrm{Chi}^{2}=0.47, \mathrm{df}=1(P=0.49), \mathrm{I}^{2}=0 \%\end{array}$}} & & & \\
\hline & & & & & & & & & $\begin{array}{ccc}1 & 1 \\
-2 & -1 & 0 \\
\text { Favours [Placebo laser] }\end{array}$ & $0 \quad 1$ & 2 \\
\hline
\end{tabular}

Fig. 13. Forest plot of stiffness between laser with physical activity and physical activity only.

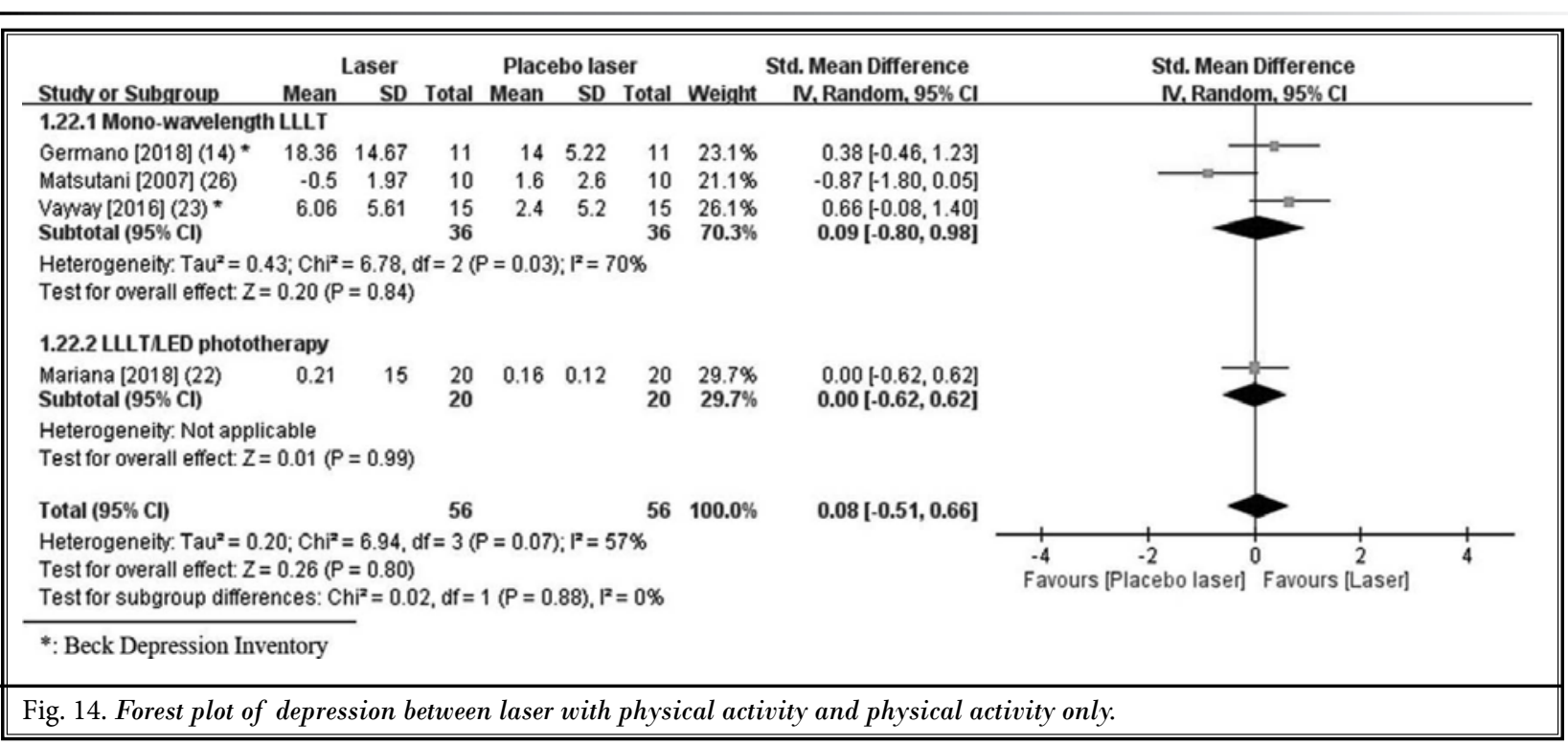

\begin{tabular}{|c|c|c|c|c|c|c|c|c|c|c|}
\hline \multirow{2}{*}{ Study or Subgroup } & \multicolumn{2}{|c|}{ Laser } & \multicolumn{4}{|c|}{ Placebo laser } & \multirow{2}{*}{\multicolumn{2}{|c|}{$\begin{array}{cc}\text { Std. Mean Difference } \\
\text { Weight } & \text { N, Random, } 95 \% \mathrm{Cl}\end{array}$}} & \multirow{2}{*}{\multicolumn{2}{|c|}{$\begin{array}{l}\text { Std. Mean Difference } \\
\text { N. Random, } 95 \% \mathrm{Cl}\end{array}$}} \\
\hline & Mean & SD & Total & Mean & SD & Total & & & & \\
\hline \multicolumn{11}{|c|}{ 1.15.1 Mono-wavelength LLIT } \\
\hline $\begin{array}{l}\text { Matsutani [2007] (26) } \\
\text { Subtotal }(95 \% \mathrm{Cl})\end{array}$ & 1 & 2.01 & $\begin{array}{l}10 \\
10\end{array}$ & 1.9 & 2.46 & $\begin{array}{l}10 \\
10\end{array}$ & $\begin{array}{l}45.1 \% \\
45.1 \%\end{array}$ & $\begin{array}{l}-0.38[-1.27,0.50] \\
-\mathbf{0 . 3 8}[-1.27,0.50]\end{array}$ & & \\
\hline \multicolumn{11}{|c|}{$\begin{array}{l}\text { Heterogeneity: Not applicable } \\
\text { Test for overall effect: } Z=0.85(P=0.40)\end{array}$} \\
\hline \multicolumn{11}{|c|}{ 1.15.2 LLILED phototherapy } \\
\hline $\begin{array}{l}\text { Mariana [2018] (22) } \\
\text { Subtotal }(95 \% \mathrm{Cl})\end{array}$ & 0.16 & 0.09 & $\begin{array}{l}20 \\
20\end{array}$ & 0.1 & 0.1 & $\begin{array}{l}20 \\
20\end{array}$ & $\begin{array}{l}54.9 \% \\
54.9 \%\end{array}$ & $\begin{array}{c}0.62[-0.02,1.25] \\
\mathbf{0 . 6 2}[-0.02,1.25]\end{array}$ & & \\
\hline \multicolumn{11}{|c|}{$\begin{array}{l}\text { Heterogeneity. Not applicable } \\
\text { Test for overall effect: } Z=1.91(P=0.06)\end{array}$} \\
\hline Total $(95 \% \mathrm{Cl})$ & & & 30 & & & 30 & $100.0 \%$ & $0.17[-0.81,1.14]$ & & \\
\hline \multicolumn{9}{|c|}{$\begin{array}{l}\text { Heterogeneity. } \text { Tau }^{2}=0.35 ; C h \mathrm{I}^{2}=3.24, \mathrm{df}=1(\mathrm{P}=0.07) ; \mathrm{I}^{2}=69 \% \\
\text { Test for overall effect. } Z=0.33(P=0.74) \\
\text { Test for subgroup differences: } \mathrm{ChI}^{2}=3.24, \mathrm{df}=1(\mathrm{P}=0.07), \mathrm{I}^{2}=69.1 \%\end{array}$} & $\begin{array}{cccc}1 & 1 & 1 & 1 \\
-4 & -2 & 0 & 2 \\
& \text { Favours [Placebo laser] } & \text { Favours [Laser] }\end{array}$ & $\begin{array}{l}1 \\
4\end{array}$ \\
\hline
\end{tabular}


opioid receptors, have been postulated to explain the analgesic effect of phototherapy (32). Other hypotheses include anti-inflammation due to a decrease in prostaglandin-2 and cyclooxygenase-2 levels $(28,32)$, proliferation and neovascularization of connective tissue cells $(33,34)$, and increase in blood flow and promotion of healing by increase in the levels of nitric oxide, a powerful vasodilator (32). A recent systematic review and meta-analysis of 18 studies suggested that LLLT effectively reduces pain in adult patients with musculoskeletal disorders; however, patients with fibromyalgia were not included in this meta-analysis (7). Furthermore, studies have indicated the beneficial role of LLLT/ LED combination in the treatment of nonspecific knee pain (35) as well as masseter and temporalis muscle pain in women with temporomandibular disorder (36).

Pain is the main symptom in patients with fibromyalgia. Some RCTs have investigated the effect of LLLT on fibromyalgia, but by using small sample sizes. Our study is the first systematic review and meta-analysis including 9 RCTs involving 325 patients to specifically evaluate the efficacy of LLLT in fibromyalgia. Our results demonstrated that LLLT provided significant improvement in FIQ score, pain severity, number of tender points, fatigue, stiffness, depression, and anxiety compared to placebo. However, when compared with pure exercise therapy, LLLT with exercise therapy did not show more benefits. On the other hand, the single RCT using LLLT/LED showed significant improvement in the above-mentioned outcomes, except for depression, when compared to placebo. When compared with patients with fibromyalgia who received exercise therapy, combined LLLT/LED phototherapy and exercise therapy had additional benefits in reducing the severity of pain, number of tender points, and fatigue.

However, this review still has some limitations, mostly because of the low-to-middle methodological quality of the selected studies (Table 2). First, most studies did not report the allocation process clearly and only blinded the patients; neither phototherapy program- mer nor outcome assessor were blinded. Considering that nearly all outcomes were subjective parameters, the above shortcomings may introduce allocation bias, performance bias, and detection bias. Second, one study used per-protocol analysis because of a $20 \%$ loss to follow-up without reporting the reasons for, or the distribution of, the loss to follow-up (26); this may have introduced attrition bias. Third, although LLLT was used in all trials, the differences in laser types, energy sources, and exposure times used in the studies may have resulted in some heterogeneity. Fourth, although patients with fibromyalgia did not take associated medications in most RCTs, patients in one trial maintained their usual pharmacological therapies (22), another trial included some patients continuing their regular medications (14), and the other 3 RCTs did not mention whether the participants were under concurrent medication (24-26); therefore, we could not clarify the separate roles of medication or phototherapy in fibromyalgia. Finally, long-term follow-up up to 6 months was only conducted in one RCT (27).

In spite of the limitations, our study is the largest systematic review and meta-analysis to evaluate the efficacy of LLLT in patients with fibromyalgia, and it has provided the most relevant available evidence on LLLT for fibromyalgia. In conclusion, our data indicate that LLLT is an emerging, noninvasive, well-tolerated treatment for fibromyalgia to relieve discomfort, particularly in patients who do not exercise regularly.

\section{Acknowledgments}

We are grateful to Mariana Moreira da Silva and all her coworkers for providing us with the raw data of their original study "Randomized, blinded, controlled trial on the effectiveness of photobiomodulation therapy and exercise training in the fibromyalgia treatment." This enriched the content of our metaanalysis and enhanced the power of evidence of LLLT. This manuscript was edited by Wallace Academic Editing. 


\section{REFERENCES}

1. Clauw DJ. Fibromyalgia: A clinical review. JAMA 2014; 311:1547-1555.

2. Watson NF, Buchwald D, Goldberg J, Noonan C, Ellenbogen RG. Neurologic signs and symptoms in fibromyalgia. Arthritis Rheum 2009; 60:2839-2844.

3. Goldenberg DL, Burckhardt C, Crofford L. Management of fibromyalgia syndrome. JAMA 2004; 292:2388-2395.

4. Okifuji A, Gao J, Bokat C, Hare BD. Management of fibromyalgia syndrome in 2016. Pain Manag 2016; 6:383-400.

5. de Andrade AL, Bossini PS, Parizotto NA. Use of low level laser therapy to control neuropathic pain: A systematic review. J Photochem Photobiol B 2016; 164:36-42.

6. Glazov G, Yelland M, Emery J. Low-level laser therapy for chronic non-specific low back pain: A meta-analysis of randomised controlled trials. Acupunct Med 2016; 34:328-341.

7. Clijsen R, Brunner A, Barbero M, Clarys $P$, Taeymans J. Effects of low-level laser therapy on pain in patients with musculoskeletal disorders: A systematic review and meta-analysis. Eur J Phys Rehabil Med 2017; 53:603-610.

8. Deana NF, Zaror C, Sandoval P, Alves N. Effectiveness of low-level laser therapy in reducing orthodontic pain: A systematic review and meta-analysis. Pain Res Manag 2017; 2017:8560652.

9. Huisstede BM, Hoogvliet P, Franke TP, Randsdorp MS, Koes BW. Carpal tunnel syndrome: Effectiveness of physical therapy and electrophysical modalities. An updated systematic review of randomized controlled trials. Arch Phys Med Rehabil 2017; 99:1623-1634.

10. Rayegani SM, Raeissadat SA, Heidari S, Moradi-Joo M. Safety and effectiveness of low-level laser therapy in patients with knee osteoarthritis: A systematic review and meta-analysis. ] Lasers Med Sci 2017; 8:S12-S19.

11. Prindeze NJ, Moffatt LT, Shupp JW. Mechanisms of action for light therapy: A review of molecular interactions. Exp Biol Med (Maywood) 2012; 237:1241-1248.

12. Higgins JP, Altman DG, Gotzsche PC, Juni P, Moher D, Oxman AD, Savovic J, Schulz KF, Weeks L, Sterne JA; Cochrane Bias Methods Group, Cochrane Statistical Methods Group. The Cochrane Collaboration's tool for assessing risk of bias in randomised trials. BMJ 2011; 343:d5928.

13. Burckhardt CS, Clark SR, Bennett RM. The fibromyalgia impact questionnaire: Development and validation. J Rheumatol 1991; 18:728-733.

14. Germano Maciel D, Trajano da Silva M, Rodrigues JA, Viana Neto JB, de Franca IM, Melo ABM, Barros da Silva TYP, de Brito Vieira WH. Low-level laser therapy combined to functional exercise on treatment of fibromyalgia: A doubleblind randomized clinical trial. Lasers Med Sci 2018; 33:1949-1959.

15. Hamilton M. A rating scale for depression. ] Neurol Neurosurg Psychiatry 1960; 23:56-62.

16. Sharp R. The Hamilton Rating Scale for Depression. Occup Med (Lond) 2015; 65:340.

17. Bell CC. DSM-IV: Diagnostic and Statistical Manual of Mental Disorders. JAMA 1994; 272:828-829.

18. Beck AT, Ward CH, Mendelson M, Mock J, Erbaugh J. An inventory for measuring depression. Arch Gen Psychiatry 1961; 4:561-571.

19. Liberati A, Altman DG, Tetzlaff J, Mulrow C, Gotzsche PC, loannidis JP, Clarke M Devereaux PJ, Kleijnen J, Moher D. The PRISMA statement for reporting systematic reviews and meta-analyses of studies that evaluate health care interventions: Explanation and elaboration. J Clin Epidemiol 2009; 62:e1-e34.

20. Hozo SP, Djulbegovic B, Hozo I. Estimating the mean and variance from the median, range, and the size of a sample. BMC Med Res Methodol 2005; 5:13.

21. DerSimonian R, Laird N. Meta-analysis in clinical trials revisited. Contemp Clin Trials 2015; 45:139-145.

22. da Silva MM, Albertini R, de Tarso $\mathrm{Ca}$ millo de Carvalho $P$, Leal-Junior ECP, Bussadori SK, Vieira SS, Bocalini DS, de Oliveira LVF, Grandinetti V, Silva JA Jr, Serra AJ. Randomized, blinded, controlled trial on effectiveness of photobiomodulation therapy and exercise training in the fibromyalgia treatment. Lasers Med Sci 2018; 33:343-351.

23. Vayvay ES, Tok D, Turgut E, Tunay VB. The effect of Laser and taping on pain, functional status and quality of life in patients with fibromyalgia syndrome: A placebo- randomized controlled clinical trial. J Back Musculoskelet Rehabil 2016; 29:77-83.
24. Ruaro JA, Frez AR, Ruaro MB, Nicolau RA. Low-level laser therapy to treat fibromyalgia. Lasers Med Sci 2014; 29:1815-1819.

25. Fernandez Garcia R, Suarez Holgado JD, Formieles Ortiz I, Zurita Ortega F, Valverde Cepeda M, Fernandez Sanchez M. [Using a laser based program in patients diagnosed with fibromyalgia]. Reumatol Clin 2011; 7:94-97.

26. Matsutani LA, Marques AP, Ferreira EA, Assumpcao A, Lage LV, Casarotto RA, Pereira CA. Effectiveness of muscle stretching exercises with and without laser therapy at tender points for patients with fibromyalgia. Clin Exp Rheumatol 2007; 25:410-415.

27. Armagan O, Tascioglu F, Ekim A, Oner C. Long-term efficacy of low level laser therapy in women with fibromyalgia: A placebo-controlled study. J Back Musculoskelet Rehabil 2006; 19:135-140.

28. Gur A, Karakoc M, Nas K, Cevik R, Sarac J, Ataoglu S. Effects of low power laser and low dose amitriptyline therapy on clinical symptoms and quality of life in fibromyalgia: A single-blind, placebocontrolled trial. Rheumatol Int 2002; 22:188-193.

29. Gur A, Karakoc M, Nas K, Cevik R, Sarac J, Demir E. Efficacy of low power laser therapy in fibromyalgia: A single-blind, placebo-controlled trial. Lasers Med Sci 2002; 17:57-61.

30. Wolfe F, Clauw DJ, Fitzcharles MA, Goldenberg DL, Katz RS, Mease P, Russell AS, Russell IJ, Winfield JB, Yunus MB. The American College of Rheumatology preliminary diagnostic criteria for fibromyalgia and measurement of symptom severity. Arthritis Care Res (Hoboken) 2010; 62:600-610.

31. Chow RT, Johnson MI, Lopes-Martins RA, Bjordal JM. Efficacy of low-level laser therapy in the management of neck pain: A systematic review and metaanalysis of randomised placebo or active-treatment controlled trials. Lancet 2009; 374:1897-1908.

32. Kingsley JD, Demchak T, Mathis R. Lowlevel laser therapy as a treatment for chronic pain. Front Physiol 2014; 5:306.

33. Bayat M, Virdi A, Rezaei F, Chien S. Comparison of the in vitro effects of low-level laser therapy and low-intensity pulsed ultrasound therapy on bony cells and stem cells. Prog Biophys $\mathrm{Mol}$ Biol 2018; 133:36-48. 
34. Chen $\mathrm{MH}$, Huang YC, Sun JS, Chao YH, Chen $\mathrm{MH}$. Second messengers mediating the proliferation and collagen synthesis of tenocytes induced by low-level laser irradiation. Lasers Med Sci 2015; 30:263-272.

35. Leal-Junior EC, Johnson DS, Saltmarche A, Demchak T. Adjunctive use of combi- nation of super-pulsed laser and lightemitting diodes phototherapy on nonspecific knee pain: Double-blinded randomized placebo-controlled trial. Lasers Med Sci 2014; 29:1839-1847.

36. Herpich CM, Leal-Junior ECP, Gomes C, Gloria I, Amaral AP, Amaral M, Politti F,
Biasotto-Gonzalez DA. Immediate and short-term effects of phototherapy on pain, muscle activity, and joint mobility in women with temporomandibular disorder: A randomized, double-blind, placebo-controlled, clinical trial. Disabil Rehabil 2018; 40:2318-2324. 